\title{
Optimal pathways involved in the treatment of sevoflurane or propofol for patients undergoing coronary artery bypass graft surgery
}

\author{
ZHEN-GUO PAN $^{1}$, XI-ZENG ZHANG ${ }^{1}$, ZHI-MEI ZHANG $^{1}$ and YUN-JIE DONG ${ }^{2}$ \\ Departments of ${ }^{1}$ Anesthesiology and ${ }^{2}$ Medical Administration, The Second People's Hospital of Liaocheng, \\ Linqing, Shandong 252600, P.R. China
}

Received June 21, 2018; Accepted February 14, 2019

DOI: $10.3892 / \mathrm{etm} .2019 .7354$

\begin{abstract}
The cardio-protection mechanisms of sevoflurane and propofol still remain unclear in patients undergoing coronary artery bypass grafting $(\mathrm{CABG})$. We designed the present study to identify the optimal pathways through integrating differential co-expressed network (DCN)-based guilt by association (GBA) principle based on the expression data of E-GEOD-4386 downloaded from EMBL-EBI. Differentially expressed genes (DEGs) were firstly identified and then DCN and sub-DCN were established. The seed pathways were predicted through GBA principle using the area under the curve (AUC) for pathway categories, and the pathway terms with AUC $>0.9$ were defined as the seed pathways. KEGG pathway analysis was applied to the DEGs based on DAVIA to detect significant pathways. The final optimal pathways were identified based on the traditional pathway analysis and network-based pathway inference approach. There were 83 common, 99 sevoflurane-specific and 4 propofol-specific DEGs in the expression profile of artial samples. Finally, 8 and 4 pathway terms having the AUC $>0.9$ were identified and determined as the seed pathways in the propofol and sevoflurane group, respectively. TNF signaling pathway, NF- $\mathrm{BB}$ signaling pathway, as well as NOD-like receptor signaling pathway were the common optimal ones in these two groups. Only the pathway of cytokine-cytokine receptor interaction was unique to sevoflurane, and no pathway was specific to propofol. Our results suggested that sevoflurane and propofol might synergistically possess some cardio-protective properties in patients undergoing CABG.
\end{abstract}

Correspondence to: Dr Yun-Jie Dong, Department of Medical Administration, The Second People's Hospital of Liaocheng, 306 Jiankang Street, Linqing, Shandong 252600, P.R. China E-mail: 176573756@qq.com

Key words: coronary artery bypass grafting, sevoflurane, propofol, differential co-expression network, pathway, guilt by association

\section{Introduction}

Coronary artery bypass grafting (CABG) has become one of the most common and effective strategies for managing coronary heart disease in the world (1). Unfortunately, CABG can induce ischemic injury, especially for patients having poor cardiac contractile function (2). Intervention methods, for example, anesthetics, before and after myocardial ischemia, enables to lessen myocardial ischemic damage to some extent $(3,4)$. Importantly, both sevoflurane and propofol are frequently used anesthetics in clinical settings $(5,6)$. Sevoflurane, as an inhalation anesthetic, plays a myocardial protective role on low risk patients treated with CABG (7). Sevoflurane has been found to exert a more remarkable effect on gene expression in patients undergoing CABG compared with propofol (8). The myocardial protection effect of sevoflurane might be achieved by downregulating platelet endothelial cell adhesion molecule-1 (9) and/or troponin I (10). On the contrary, propofol has performed better in cardiovascular instability, severe ischemia or acute/urgent surgery patients (11). Additionally, intravenous anesthetics propofol has been implicated to attenuate myocardial lipid peroxidation and systemic inflammation in CABG surgery $(12,13)$. Growing evidence has demonstrated that sevoflurane as well as propofol exert important functions in cardio protection. Nevertheless, the potential cardio-protection mechanisms of these anaesthetics still remain unclear, and which anesthetic is appropriate also needs to be investigated.

Microarray profiles provide a basic starting point in the detection of mechanistic causes. One standard approach is extracting differentially expressed genes (DEGs). However, this approach provides only limited information on the biological role of the DEGs. Moreover, there is frequently little overlap between microarray studies $(14,15)$, but pathway analysis can conquer the weaknesses of the current single-locus approaches. It has been demonstrated that pathway-based analysis has the ability of enhancing power and robustness, as well as extracting biological interaction among gene pairs $(16,17)$. Investigating biological pathways based on system biology techniques can provide comprehensive insights into the components of pathways, thereby facilitating the inference of novel targets for diseases (18). It has been demonstrated that network of gene-gene functional interactions is useful 
in predicting biological functions $(19,20)$. There are many approaches to predict function based on gene-gene networks. Significantly, the most common method used in the studies is some variation of the guilt by association (GBA) $(21,22)$. It has been suggested that GBA can predict pathways in all kinds of biological networks such as gene co-expression network (19).

Hence, in the present study, in order to provide the foundation to select the appropriate anesthetic, network-based method and GBA principle were used to further identify the optimal pathways using the known pathway data and microarray profile. E-GEOD-4386 data were used to identify DEGs. Then, a differential co-expressed network (DCN) was constructed using DEGs, and sub-DCN was extracted relying on the weight values which were computed using Spearman's correlation coefficient (SCC). The KEGG pathways for CABG were collected on the basis of the known confirmed database as well as DEGs and the seed pathways were predicted through GBA principle according to the area under the curve (AUC) of pathway categories, and the pathway terms with AUC $>0.9$ were defined as the seed pathways. KEGG pathway enrichment analysis was conducted for the DEGs based on the DAVID tool to detect the significant pathways. The final optimal pathways were identified based on the traditional pathway analysis and network-based pathway inference approach. These pathways may be helpful for the appropriate selection of propofol or sevoflurane, thus improving the clinical outcomes of patients undergoing $\mathrm{CABG}$ surgery.

\section{Materials and methods}

Gene expression profile and pre-treatment. The expression data of E-GEOD-4386 was downloaded from the A-AFFY-44 - Affymetrix GeneChip Human Genome U133 Plus 2.0 platform of ArrayExpress database (http://www.ebi. ac.uk/arrayexpress/). The E-GEOD-4386 dataset includes data of 40 samples: 10 patients who underwent CABG with intravenous anesthetic propofol treatment, 10 patients who underwent $\mathrm{CABG}$ with anesthetic gas sevoflurane treatment and 20 baseline samples (23). All the patients underwent the same procedure except for the anesthesia. The baseline samples were of the same patients before CABG. The atrial samples were obtained prior to as well as after CABG to investigate gene expression. The microarray profiling of the 40 atrial samples were downloaded to further detect DEGs.

Before analysis, E-GEOD-4386 was pre-processed, including background correction using robust multi-array average (RMA) (24), quartile normalization (25), and perfect match (PM)/mismatch (MM) correction by means of MAS 5.0 package (26). Finally, probe data were mapped to human gene symbols using annotate package (27). Finally, 20,514 genes were obtained for further investigation.

Identification of DEGs. The LIMMA package (28) with t-test was used in our work to compare the gene data before and after sevoflurane or propofol treatment to further achieve DEGs between two samples. SPSS 17.0 (SPSS, Inc., Chicago, IL, USA) was used to process the raw data through $\log 2$ transformation. Then, multiple test was used to adjust the original P-values relying on Benjamini \& Hochberg (29) according to false discovery rate (FDR). The cut-off criteria of DEGs were regarded to be FDR $<0.05$ as well as llog fold change $(\mathrm{FC}) \mid \geq 0.5$.

Establishment of DCN. After identification of DEGs, the construction of DCN was performed based on DEGs using Cytoscape software. Then, SCC was applied to measure the co-expressed strength of each edge in the DCN. In the current study, the absolute value of SCC of one edge was defined as the weight of the corresponding interaction. The greater the weight score was, the more relevant the interaction was to the disease or clinical effect. Hence, the edges having weight scores $>0.8$ were selected to establish the sub-DCN which was also visualized using Cytoscape software.

KEGG annotation for DEGs. KEGG provides a reference knowledge base for better understanding the biological processes. In the current study, overall 300 background pathways covering 6,919 genes were collected from KEGG database. Next, the identified DEGs were mapped to the 300 pathway terms to extract the DEG-related pathways. Finally, the pathway slim set was required in propofol treatment, and sevoflurane treatment groups, consisting of 87 DEGs and 64 pathways in propofol treatment, and 182 DEGs as well as 84 pathways in sevoflurane group.

Seed pathways using 'GBA' prediction. Then, GBA method was employed for the DCN to further predict significant biological pathways in patients undergoing $\mathrm{CABG}$ procedure. In detail, 3-fold cross-validation was utilized to obtain a gene list sorted using the ranked scoring in the DCN as to how these genes participated in the known pathway categories. With regard to every gene of the DCN, we mapped all neighbouring genes of this gene to each pathway term, and we then calculated the multifunctionality (MF) value for each gene enriched in the pathway term.

Afterwards, we calculated the AUC value for each pathway category using support vector machine (SVM), and the mean value of the AUC across all pathway categories was obtained. Thus, the pathway terms were ranked relying on the AUC scores. In the literature related to the gene functions, AUC values $>0.7$ were considered good (30). In this study, the pathway terms of AUC $>0.9$ were regarded to be the seed pathways.

Pathway analyses for DEGs using DAVID. KEGG provides a reference knowledge base for better understanding biological processes. DAVID (http://david.abcc.ncifcrf.gov/) is an analytical tool used to analyze a large number of genes (31). To further explore the biological functions of DEGs, DAVID was utilized to conduct the traditional pathway analysis using the Expression Analysis Systematic Explorer (EASE) test (32) based on KEGG pathway database. The significant pathways were extracted when FDR was set at 0.001 , and gene count $>5$.

\section{Results}

Sevoflurane influences more DEGs compared with propofol. Before DCN construction, DEGs in propofol and sevoflurane treatment were screened out. When the threshold was set at 


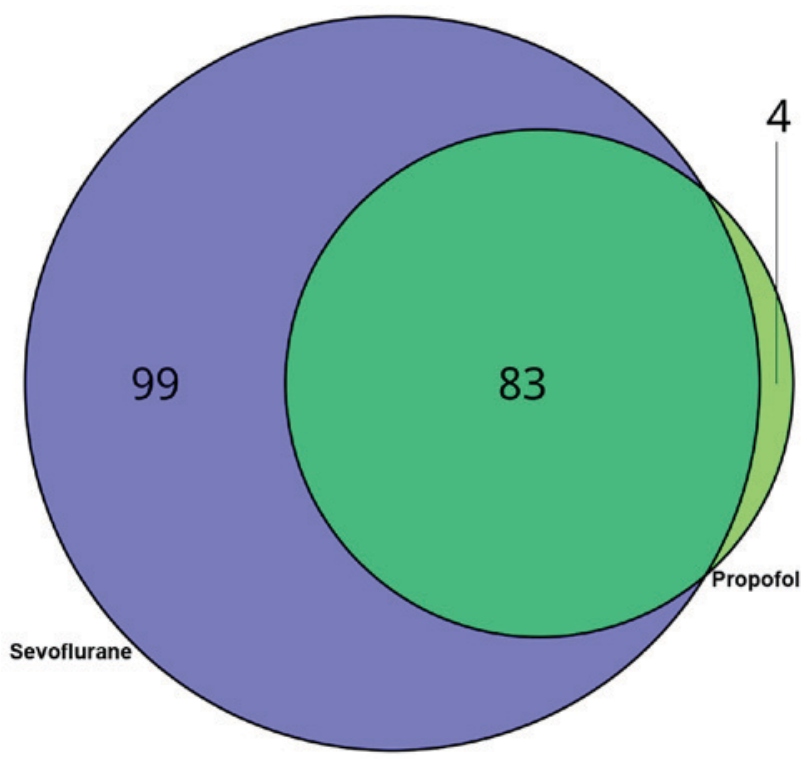

Figure 1. Venn diagram demonstrating the count of differentially expressed genes (DEGs) between propofol and sevoflurane groups.

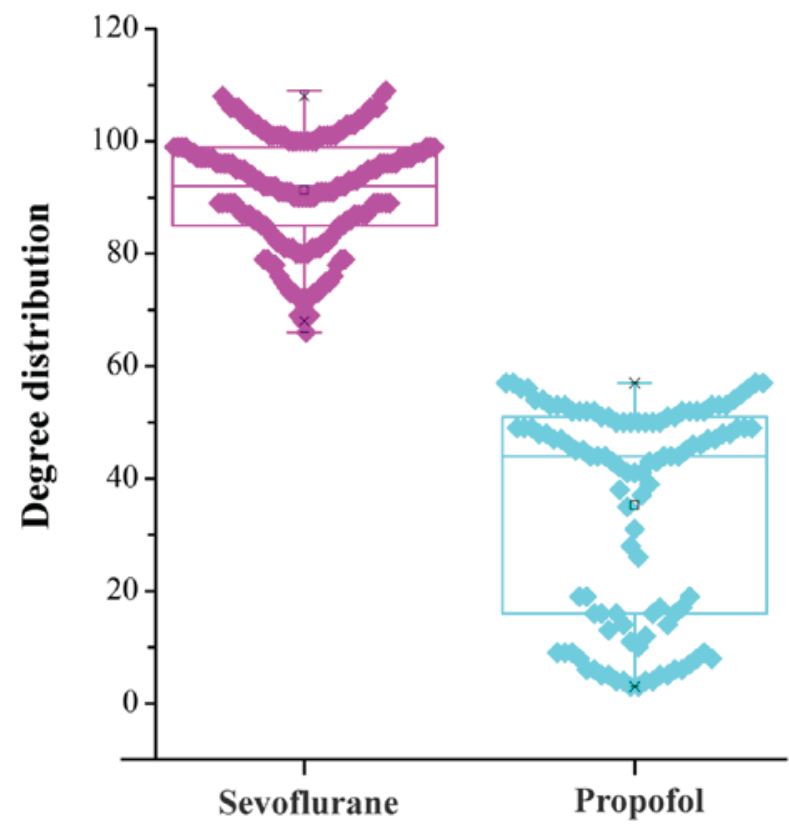

Figure 2. Degree distribution of the nodes in the differentially co-expressed network (DCN) between the two groups.
FDR $<0.05$ and $\mid \log \mathrm{FCl} \geq 0.5$, compared with the baseline group, there were respectively 87 and 182 DEGs in patients treated with propofol and sevoflurane (Fig. 1). The expression levels of the DEGs in propofol and sevoflurane groups are available in Tables SI and SII, respectively (supplementary material). Then, it was found that 83 DEGs were common between the two groups. Significantly, 99 DEGs were unique to sevoflurane treatment, while 4 DEGs were specific to the patients who received propofol treatment.

To further reveal the DEG-biological activities, a DCN was established for propofol and sevoflurane groups relying on the above-identified DEGs. There were 87 nodes within the DCN of propofol group and 182 DEGs in the DCN of the sevoflurane group. These demonstrated that all DEGs were mapped to the DCN. In the network, degree can explain the network structure. Thus, we analyzed the topological degree for each node in the DCN, and the degree distribution for each node is shown in Fig. 2. The degrees in sevoflurane group were higher than those in propofol group. Then, SCC was utilized to calculate the weight score of each edge, and the interactions having weight values $>0.8$ were selected to build the sub-DCN (Fig. 3). Overall, 81 nodes and 728 interactions were involved in the sub-DCN of the propofol group, and 175 nodes and 3,286 interactions were in the sub-DCN of the sevoflurane group.

Seed pathways using GBA prediction. The AUC distribution for pathway terms is displayed in Fig. 4. The AUC for most pathways ranged from 0.4 to 0.8 in these two groups. Together, 38 and 28 pathway terms were respectively identified in the propofol and sevoflurane groups based on AUC >0.7 Significantly, among these pathways, the AUC value of 8 pathways in the propofol group and 4 pathways in the sevoflurane group was higher than 0.9 , and these pathways were determined as the seed pathways. Specific information on the seed pathways is listed in Table I.

Pathway enrichment analysis for DEGs. Relying on FDR $<0.001$ as well as gene count $>5,8$ significant pathways were identified in the propofol group, and 10 significant pathways were detected in the sevoflurane group. The list of differential pathways in the two groups is shown in Table II. From the Table, we found that most of the differential pathways were the same in these two groups $(\mathrm{NF}-\kappa \mathrm{B}$ signaling pathway,

Table I. The seed pathways in the propofol and sevoflurane groups.

\begin{tabular}{|c|c|c|c|}
\hline Propofol-specific pathways & AUC & Sevoflurane-specific pathways & AUC \\
\hline hsa04064:NF- $\kappa \mathrm{B}$ signaling pathway & 0.9921 & hsa04060:Cytokine-cytokine receptor interaction & 0.9315 \\
\hline hsa04623-Cytosolic DNA-sensing & 0.9921 & hsa04064:NF-кB signaling pathway & 0.9249 \\
\hline hsa05321-inflammatory bowel disease & 0.9922 & hsa04621:NOD-like receptor signaling pathway & 0.9181 \\
\hline hsa05332- graft-versus-host disease & 0.9843 & hsa04668:TNF signaling pathway & 0.9111 \\
\hline hsa05133-Pertussis & 0.9715 & & \\
\hline hsa05203-Viral carcinogenesis & 0.9325 & & \\
\hline hsa04621-NOD-like receptor signaling pathway & 0.9104 & & \\
\hline hsa04668:TNF signaling pathway & 0.9002 & & \\
\hline
\end{tabular}



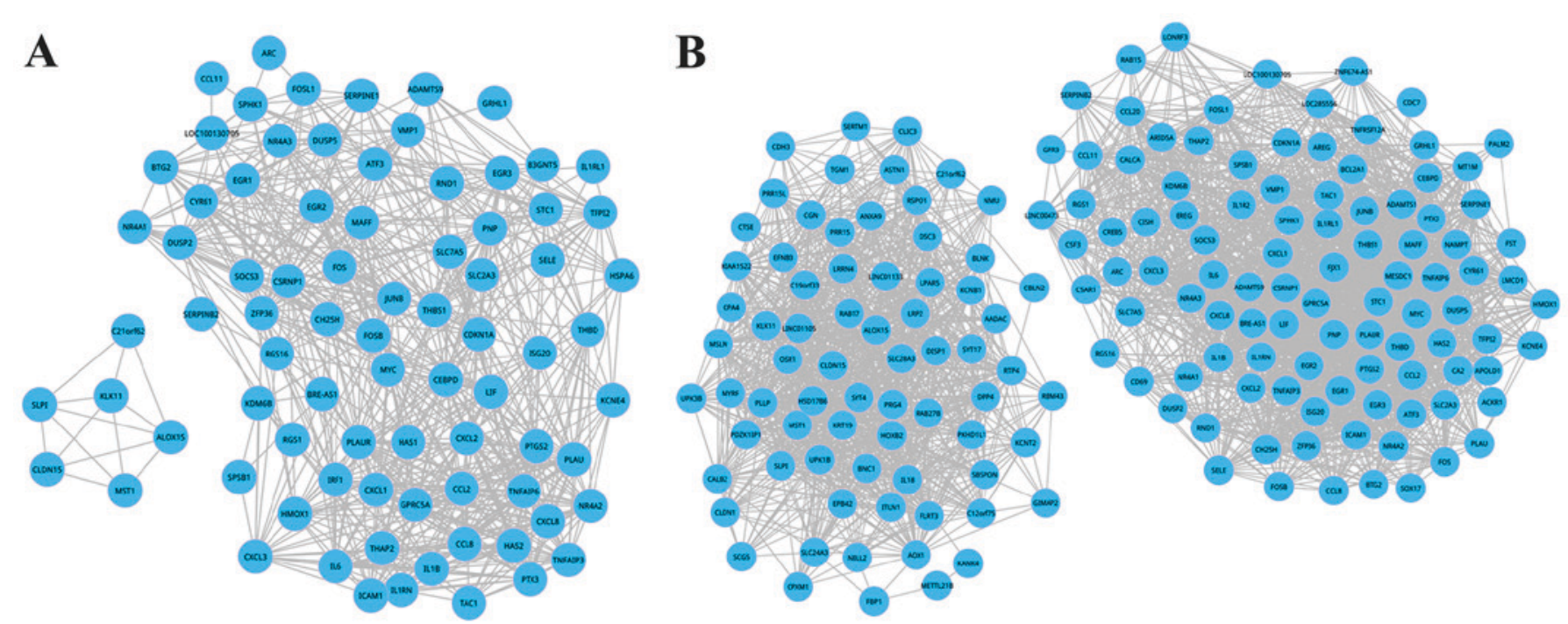

Figure 3. Sub-DCN establishment for the two groups according to the weight value $>0.8$. (A) Sub-DCN composition for propofol group. (B) Sub-DCN composition for sevoflurane group.
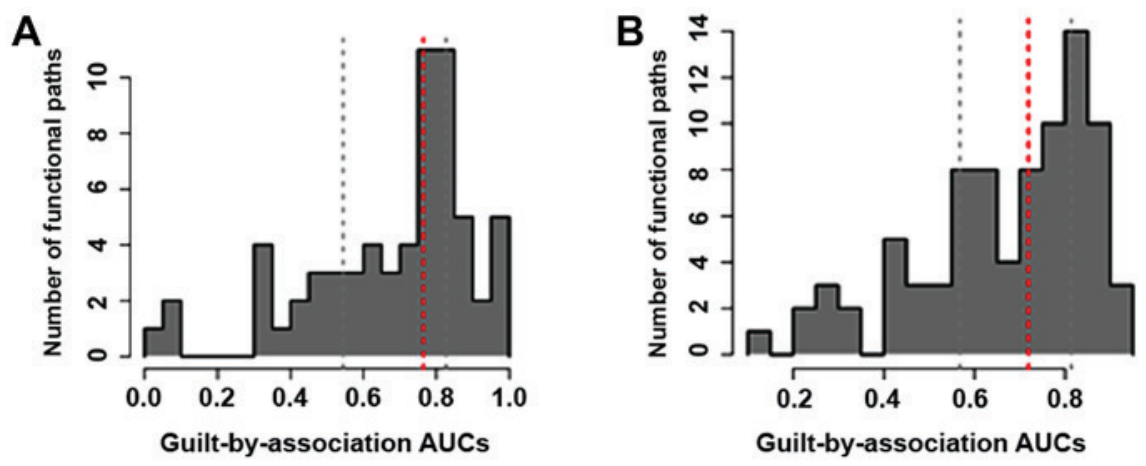

Figure 4. Pathway prediction relying on guilt by association (GBA). Histogram of AUCs across all pathway terms which are gained on the basis of a single list constructed from a number of co-expression genes. (A) The AUC distribution for pathway terms of propofol group. (B) The AUC distribution for pathway terms of sevoflurane group.

Table II. List of the differential pathways in the propofol and sevoflurane groups.

\begin{tabular}{|c|c|c|c|c|c|}
\hline Propofol-specific differential pathways & FDR & $\begin{array}{l}\text { Gene } \\
\text { count }\end{array}$ & Sevoflurane-specific pathways & FDR & $\begin{array}{l}\text { Gene } \\
\text { count }\end{array}$ \\
\hline hsa04668:TNF signaling pathway & 2.04E-13 & 14 & hsa04668:TNF signaling pathway & $4.38 \mathrm{E}-13$ & 16 \\
\hline hsa05144:Malaria & $1.03 \mathrm{E}-06$ & 7 & hsa05144:Malaria & 2.65E-09 & 10 \\
\hline hsa05134:Legionellosis & $1.85 \mathrm{E}-06$ & 7 & hsa05134:Legionellosis & $2.86 \mathrm{E}-05$ & 7 \\
\hline hsa05132:Salmonella infection & 2.32E-05 & 7 & hsa05132:Salmonella infection & $3.71 \mathrm{E}-05$ & 8 \\
\hline hsa05166:HTLV-I infection & $6.40 \mathrm{E}-05$ & 10 & $\begin{array}{l}\text { hsa04060:Cytokine-cytokine } \\
\text { receptor interaction }\end{array}$ & 4.76E-05 & 12 \\
\hline hsa04064:NF-кB signaling pathway & $3.48 \mathrm{E}-04$ & 6 & hsa04064:NF-кB signaling pathway & $5.04 \mathrm{E}-05$ & 8 \\
\hline hsa05323:Rheumatoid arthritis & 3.67E-04 & 6 & hsa05323:Rheumatoid arthritis & $5.42 \mathrm{E}-05$ & 8 \\
\hline \multirow[t]{2}{*}{ hsa04621:NOD-like receptor signaling pathway } & 5.84E-04 & 7 & $\begin{array}{l}\text { hsa04621:NOD-like receptor } \\
\text { signaling pathway }\end{array}$ & $3.60 \mathrm{E}-04$ & 6 \\
\hline & & 5 & hsa05143:African trypanosomiasis & 4.80E-04 & 6 \\
\hline
\end{tabular}

NOD-like receptor signaling pathway and TNF signaling pathway). Only the pathway of cytokine-cytokine receptor interaction, and African trypanosomiasis were unique to sevoflurane. However, no pathway was unique to propofol. 
Identifying the optimal pathways. The final optimal pathways were identified based on the traditional pathway analysis and network-based pathway inference approach. Finally, 3 optimal pathways were identified in the propofol group, including $\mathrm{NF}-\kappa \mathrm{B}$ signaling pathway, TNF signaling pathway, and NOD-like receptor signaling pathway. Moreover, 4 optimal pathways were identified in the sevoflurane group, including cytokine-cytokine receptor interaction, NOD-like receptor signaling pathway, $\mathrm{NF}-\kappa \mathrm{B}$ signaling pathway, and TNF signaling pathway. Based on these results, we observed that NOD-like receptor signaling pathway, $N F-\kappa B$ signaling pathway, and TNF signaling pathway is the common optimal one in these two groups. Cytokine-cytokine receptor interaction was unique to sevoflurane.

\section{Discussion}

Previous studies have reported that sevoflurane and propofol are the two most common and effective anesthetic agents in CABG surgery (33). Nevertheless, the underlying cardioprotection mechanisms of the two anaesthetics still remain unclear, and which anesthetic is appropriate also need to be investigated. Compared to studying single gene biomarkers, investigating biological functions appears more promising in understanding the disease-related insights (34). Network analysis has been broadly utilized in many diseases to comprehend the biological processes of diseases, and to further obtain clinical insights (35). In recent years, GBA method has been proposed to predict gene functions based on the indirect connections $(36,37)$. Nevertheless, integration of gene-related pathway prediction and network modeling are sparse. Therefore, herein, GBA method combined with DCN-based analysis were used to further investigate the optimal pathways for patients treated by CABG surgery plus propofol or sevoflurane management, relying on the known KEGG pathway data and microarray profile. In the current work, 83 common, 99 sevoflurane-specific as well as 4 propofol-specific DEGs from the expression profile of atrial samples were found.

Finally, there were 8 and 4 pathway terms having AUC $>0.9$ and these pathways were considered as the seed pathways. Traditional pathway analysis demonstrated that most of the differential pathways were the same in these two groups. Only cytokine-cytokine receptor interaction was unique to sevoflurane, and no pathway was specific to propofol.

The pathways of NF- $\mathrm{B}$ signaling pathway, TNF signaling pathway, and NOD-like receptor signaling were common in the protofol and sevoflurane groups, which were associated with immune responses. Myocardial ischaemia activates $\mathrm{NF}-\kappa \mathrm{B}$ causing the generation of inflammatory mediators such as TNF- $\alpha$ and IL-1 (38). Furthermore, sevoflurane has been demonstrated to reduce the levels of inflammatory cytokines and to inhibit NF- $\kappa \mathrm{B}$ activation (39). Moreover, inhibiting $\mathrm{NF}-\kappa \mathrm{B}$ has been suggested to provide the protection against myocardial ischaemia in sevoflurane preconditioning (40). Another study has reported that propofol can effectively attenuate the inflammation through $\mathrm{NF}-\kappa \mathrm{B}$ signal pathway (41). NOD-like receptor is involved in the innate immunity and inflammation $(42,43)$. It has been suggested that cardiac surgery produces a systemic inflammatory response, which was caused by surgical trauma (44). Consequently, understanding the inflammatory condition of patients prior to CABG surgery is crucially significant to reduce the postoperative complications.

The pathway of cytokine-cytokine receptor interaction was unique to sevoflurane in our study. Another study also has demonstrated that the pathway associated with cytokinecytokine receptor interaction is unique to sevoflurane (8). Cytokines are a group of molecules that transmit intercellular signals, and they induce responses through binding to specific receptors on the cell surface (45). Moreover, injured endothelial cells give rise to cytokines (for example, interleukin) that stimulate the expression of adhesion proteins and cell adhesion molecules (46). It has been implicated that elevated level of IL-8 is related to an increased risk of coronary artery disease (47). Accordingly, this result further meant that the anesthetic sevoflurane might provide the patients with more protection in inflammatory diseases.

Of course, there were several limitations in this study. Limited samples were used to predict the pathway biomarkers, which might result in biased estimates. Moreover, given that only bioinformatics methods were used in our study, the conclusions have not been confirmed based on any lab experiment. Despite these shortcomings, our study provided important implications for the molecular mechanisms of cardio-protection of sevoflurane and propofol in $\mathrm{CABG}$ surgery, but a further study is needed to validate our findings relying on lab techniques.

In the current work, the data were recruited from the E-GEOD-4386 which was produced by Lucchinetti et al (23). Based on the study of Lucchinetti et al, DEGs between the two groups were detected in our study, and pathway enrichment analyses were also conducted to examine the underlying mechanism of the two anaesthetic agents. Nevertheless, certain discrepancies also exist. Few studies have identified potential pathways based on the traditional pathway analysis and network-based pathway inference approach using AUC indicator. According to the study of Lucchinetti et al, further analyses relying on bioinformatics were implemented in the current study, including the construction of DCN, seed pathway identification, and AUC calculation. Although Bu et al (48) and Li et al (8) have also analyzed the effect of sevoflurane and propofol on gene expression based on the E-GEOD-4386 dataset, the methods used and the outcomes in their research are different from our study. A GBA method combined with DCN-based analysis was used to identify optimal pathways in our study, while Bu et al (48) used module topological analysis to evaluate significant pathway-related modules and Li et al (8) only identified the DEGs and performed GO and KEGG pathway enrichment analyses for DEGs. Compared with the previous studies, some novel seed pathways were found in our study, such as $\mathrm{NF}-\kappa \mathrm{B}$ signaling pathway, inflammatory bowel disease, graft-versus-host disease and TNF signaling pathway. Hence, our results provide new insights into the understanding of cardio-protection mechanisms of sevoflurane and propofol.

Taken together, sevoflurane and propofol might synergistically decrease myocardial reperfusion injury of patients treated by $\mathrm{CABG}$, because similarity and particularity were all found in the pathway alterations caused by propofol and sevoflurane. Our present study deepens the understanding of cardio protective mechanism of sevoflurane and propofol. The optimal pathways in our study may be helpful for 
the appropriate selection of propofol or sevoflurane, thus promoting improvement in the clinical outcomes of patients undergoing CABG surgery. Further efforts will be made to investigate the underlying cardio-protection mechanisms of anaesthetics in animal models.

\section{Acknowledgements}

Not applicable.

\section{Funding}

No funding was received.

\section{Availability of data and materials}

The datasets used and/or analyzed during the current study are available from the corresponding author on reasonable request.

\section{Authors' contributions}

ZGP and YJD designed this research. ZGP and XZZ collected the data and prepared the figures. ZGP and ZMZ analyzed the data. ZGP wrote the manuscript. YJD contributed substantially to its revision. All the authors have read and approved this manuscript.

\section{Ethics approval and consent to participate}

Not applicable.

\section{Patient consent for publication}

Not applicable.

\section{Competing interests}

The authors declare that they have no competing interests.

\section{References}

1. Rezaianzadeh A, Maghsoudi B, Tabatabaee H, Keshavarzi S, Bagheri Z, Sajedianfard J, Gerami H and Rasouli J: Factors associated with extubation time in coronary artery bypass grafting patients. PeerJ 3: e1414, 2015.

2. Selvanayagam JB, Petersen SE, Francis JM, Robson MD, Kardos A, Neubauer S and Taggart DP: Effects of off-pump versus on-pump coronary surgery on reversible and irreversible myocardial injury: A randomized trial using cardiovascular magnetic resonance imaging and biochemical markers. Circulation 109: 345-350, 2004.

3. Varadarajan SG, An J, Novalija E and Stowe DF: Sevoflurane before or after ischemia improves contractile and metabolic function while reducing myoplasmic $\mathrm{Ca}(2+)$ loading in intact hearts. Anesthesiology 96: 125-133, 2002.

4. Frässdorf J, De Hert S and Schlack W: Anaesthesia and myocardial ischaemia/reperfusion injury. Br J Anaesth 103: 89-98, 2009.

5. Hellström J, Öwall A, Bergström J and Sackey PV: Cardiac outcome after sevoflurane versus propofol sedation following coronary bypass surgery: A pilot study. Acta Anaesthesiol Scand 55: 460-467, 2011.

6. Wang J, Zheng H, Chen CL, Lu W and Zhang YQ: Sevoflurane at 1 MAC provides optimal myocardial protection during off-pump CABG. Scand Cardiovasc J 47: 175-184, 2013.
7. Lin E and Symons JA: Volatile anaesthetic myocardial protection: A review of the current literature. HSR Proc Intensive Care Cardiovasc Anesth 2: 105-109, 2010.

8. Li H, Cang J and Zhang X: Sevoflurane exerts a more marked influence compared with propofol on gene expression in patients undergoing coronary artery bypass graft surgery. Exp Ther Med 11: 448-454, 2016.

9. Garcia C, Julier K, Bestmann L, Zollinger A, von Segesser LK, Pasch T, Spahn DR and Zaugg M: Preconditioning with sevoflurane decreases PECAM-1 expression and improves one-year cardiovascular outcome in coronary artery bypass graft surgery. Br J Anaesth 94: 159-165, 2005.

10. Yao YT and Li LH: Sevoflurane versus propofol for myocardial protection in patients undergoing coronary artery bypass grafting surgery: A meta-analysis of randomized controlled trials. Chin Med Sci J 24: 133-141, 2009.

11. Jakobsen CJ, Berg H, Hindsholm KB, Faddy N and Sloth E: The influence of propofol versus sevoflurane anesthesia on outcome in 10,535 cardiac surgical procedures. J Cardiothorac Vasc Anesth 21: 664-671, 2007.

12. Sayin MM, Özatamer O, Taşöz R, Kilinç K and Ünal N: Propofol attenuates myocardial lipid peroxidation during coronary artery bypass grafting surgery. Br J Anaesth 89: 242-246, 2002.

13. Corcoran TB, Engel A, Sakamoto H, O'Shea A, O'CallaghanEnright S and Shorten GD: The effects of propofol on neutrophil function, lipid peroxidation and inflammatory response during elective coronary artery bypass grafting in patients with impaired ventricular function. Br J Anaesth 97: 825-831, 2006.

14. Louwen F, Muschol-Steinmetz C, Reinhard J, Reitter A and Yuan J: A lesson for cancer research: Placental microarray gene analysis in preeclampsia. Oncotarget 3: 759-773, 2012.

15. Subramanian A, Tamayo P, Mootha VK, Mukherjee S, Ebert BL, Gillette MA, Paulovich A, Pomeroy SL, Golub TR, Lander ES, et al: Gene set enrichment analysis: a knowledgebased approach for interpreting genome-wide expression profiles. Proc Natl Acad Sci USA 102: 15545-15550, 2005.

16. Tilford CA and Siemers NO: Gene set enrichment analysis. Methods Mol Biol 563: 99-121, 2009.

17. Curtis RK, Oresic M and Vidal-Puig A: Pathways to the analysis of microarray data. Trends Biotechnol 23: 429-435, 2005.

18. Qin T: Inferring biological knowledge of pathways from an ontology fingerprint-derived gene network. PhD dissertation, Medical University of South Carolina. Publication no. AAI3569828, Charleston, SC, USA, 2012.

19. Peña-Castillo L, Tasan M, Myers CL, Lee H, Joshi T, Zhang C, Guan Y, Leone M, Pagnani A, Kim WK, et al: A critical assessment of Mus musculus gene function prediction using integrated genomic evidence. Genome Biol 9 (Suppl 1): S2, 2008.

20. Mostafavi S, Ray D, Warde-Farley D, Grouios C and Morris Q: GeneMANIA: A real-time multiple association network integration algorithm for predicting gene function. Genome Biol 9 (Suppl 1): S4, 2008.

21. Sharan R, Ulitsky I and Shamir R: Network-based prediction of protein function. Mol Syst Biol 3: 88-88, 2007.

22. Zuberi K, Franz M, Rodriguez H, Montojo J, Lopes CT, Bader GD and Morris Q: GeneMANIA prediction server 2013 update. Nucleic Acids Res 41: W115-W122, 2013.

23. Lucchinetti E, Hofer C, Bestmann L, Hersberger M, Feng J, Zhu M, Furrer L, Schaub MC, Tavakoli R, Genoni M, et al: Gene regulatory control of myocardial energy metabolism predicts postoperative cardiac function in patients undergoing off-pump coronary artery bypass graft surgery: Inhalational versus intravenous anesthetics. Anesthesiology 106: 444-457, 2007.

24. Irizarry RA, Hobbs B, Collin F, Beazer-Barclay YD, Antonellis KJ, Scherf U and Speed TP: Exploration, normalization, and summaries of high density oligonucleotide array probe level data. Biostatistics 4: 249-264, 2003.

25. Bolstad BM,Irizarry RA, Åstrand M and Speed TP: A comparison of normalization methods for high density oligonucleotide array data based on variance and bias. Bioinformatics 19: 185-193, 2003.

26. Pepper SD, Saunders EK, Edwards LE, Wilson CL and Miller CJ: The utility of MAS5 expression summary and detection call algorithms. BMC Bioinformatics 8: 273, 2007.

27. Zhu LJ, Gazin C, Lawson ND, Pagès H, Lin SM, Lapointe DS and Green MR: ChIPpeakAnno: A bioconductor package to annotate ChIP-seq and ChIP-chip data. BMC Bioinformatics 11: $237,2010$. 
28. Smyth GK: Limma: linear models for microarray data. In: Bioinformatics and Computational Biology Solutions Using R and Bioconductor. Gentleman R, Carey VJ, Huber W, Irizarry RA and Dudoit S (eds). Statistics for Biology and Health. Springer, New York, NY, pp397-420, 2005.

29. Benjamini Y, Drai D, Elmer G, Kafkafi N and Golani I: Controlling the false discovery rate in behavior genetics research. Behav Brain Res 125: 279-284, 2001.

30. Gillis $\mathrm{J}$ and Pavlidis P: The role of indirect connections in gene networks in predicting function. Bioinformatics 27: 1860-1866, 2011.

31. Huang W, Sherman BT and Lempicki RA: Systematic and integrative analysis of large gene lists using DAVID bioinformatics resources. Nat Protoc 4: 44-57, 2009.

32. Ford G, Xu Z, Gates A, Jiang J and Ford BD: Expression Analysis Systematic Explorer (EASE) analysis reveals differential gene expression in permanent and transient focal stroke rat models. Brain Res 1071: 226-236, 2006.

33. Neilsen PM, Cheney KM, Li CW, Chen JD, Cawrse JE, Schulz RB, Powell JA, Kumar R and Callen DF: Identification of ANKRD11 as a p53 coactivator. J Cell Sci 121: 3541-3552, 2008.

34. Ponomarev I, Wang S, Zhang L, Harris RA and Mayfield RD: Gene coexpression networks in human brain identify epigenetic modifications in alcohol dependence. J Neurosci 32: 1884-1897, 2012.

35. Doig TN, Hume DA, Theocharidis T, Goodlad JR, Gregory CD and Freeman TC: Coexpression analysis of large cancer datasets provides insight into the cellular phenotypes of the tumour microenvironment. BMC Genomics 14: 469, 2013.

36. Chua HN, Sung WK and Wong L: Exploiting indirect neighbours and topological weight to predict protein function from proteinprotein interactions. Bioinformatics 22: 1623-1630, 2006.

37. Yip AM and Horvath S: Gene network interconnectedness and the generalized topological overlap measure. BMC Bioinformatics 8: 22, 2007

38. Bowie AG and O'Neill LA: Vitamin C inhibits NF-kappa B activation by TNF via the activation of $\mathrm{p} 38$ mitogen-activated protein kinase. J Immunol 165: 7180-7188, 2000.

39. Zhong $\mathrm{C}$, Zhou Y and Liu H: Nuclear factor kappaB and anesthetic preconditioning during myocardial ischemia-reperfusion. Anesthesiology 100: 540-546, 2004.
40. Konia MR, Schaefer S and Liu H: Nuclear factor-[kappa]B inhibition provides additional protection against ischaemia/ reperfusion injury in delayed sevoflurane preconditioning. Eur J Anaesthesiol 26: 496-503, 2009.

41. Tian Y, Guo S, Guo Y and Jian L: Anesthetic propofol attenuates apoptosis, $\mathrm{A} \beta$ accumulation, and inflammation induced by sevoflurane through NF- $\mathrm{B}$ pathway in human neuroglioma cells. Cell Mol Neurobiol 35: 891-898, 2015.

42. Chen G, Shaw MH, Kim YG and Nuñez G: NOD-like receptors: Role in innate immunity and inflammatory disease. Annu Rev Pathol 4: 365-398, 2009.

43. Kanneganti TD, Lamkanfi M and Núñez G: Intracellular NOD-like receptors in host defense and disease. Immunity 27 : 549-559, 2007.

44. Levy JH and Tanaka KA: Inflammatory response to cardiopulmonary bypass. Ann Thorac Surg 75: S715-S720, 2003.

45. Ozaki K and Leonard WJ: Cytokine and cytokine receptor pleiotropy and redundancy. J Biol Chem 277: 29355-29358, 2002

46. Auer J, Weber T, Berent R, Lassnig E, Lamm G and Eber B: Genetic polymorphisms in cytokine and adhesion molecule genes in coronary artery disease. Am J Pharmacogenomics 3: 317-328, 2003.

47. Boekholdt SM, Peters RJ, Hack CE, Day NE, Luben R, Bingham SA, Wareham NJ, Reitsma PH and Khaw KT: IL-8 plasma concentrations and the risk of future coronary artery disease in apparently healthy men and women: The EPICNorfolk prospective population study. Arterioscler Thromb Vasc Biol 24: 1503-1508, 2004.

48. Bu X, Wang B, Wang Y, Wang Z, Gong C, Qi F and Zhang C: Pathway-related modules involved in the application of sevoflurane or propofol in off-pump coronary artery bypass graft surgery. Exp Ther Med 14: 97-106, 2017.

This work is licensed under a Creative Common Attribution-NonCommercial-NoDerivatives 4.0 International (CC BY-NC-ND 4.0) License. 